\title{
Estimation of Ready Queue Processing Time using Efficient Factor Type Estimator (E-F-T) in Multiprocessor Environment
}

\author{
${ }^{1}$ D. Shukla \\ Deptt. of Mathematics and Statistics \\ Dr.H.S.Gour Central University \\ Sagar, (M.P), INDIA
}

\author{
${ }^{2}$ Anjali Jain \\ Deptt. of Computer Science and \\ Applications \\ Dr.H.S.Gour Central University \\ Sagar ,(M.P.), INDIA
}

\begin{abstract}
The ready queue processing time estimation problem appears when many processes remain in the ready queue after the sudden failure. The system manager has to decide immediately how much further time is required to process remaining jobs in the ready queue. In lottery scheduling, this prediction is possible with the help of sampling techniques. Ratio method, existing in literature of sampling, was previously used by authors to predict the time required provided highly correlated source of auxiliary information is available and used.

This paper suggests two new estimation methods which are compared in terms of estimating the total processing time. Under large sample approximation, the bias and m.s.e of proposed estimators have been obtained in the set up of random sampling applicable to lottery scheduling. Performance of both is compared in terms of mean squared error. The confidence intervals are calculated for the estimate and they provide strong numerical support to the theoretical findings.
\end{abstract}

\section{Keywords}

Lottery Scheduling, Efficient-Factor-Type Estimator, Bias, Mean Squared Error (M.S.E), Variance, Confidence Intervals.

\section{INTRODUCTION}

Suppose there are $\mathrm{k}$ processors in a multiprocessor and multiuser environment and a large number of processes, say $\mathrm{N}$, are in a waiting queue. The CPU scheduler adopts lottery scheduling procedure to choose randomly any $\mathrm{n}$ processes from the waiting queue $(\mathrm{n}<\mathrm{N})$ and allocates to $\mathrm{k}$ processors $(\mathrm{k}<\mathrm{N})$ in sequential manner. Lottery scheduling is different from basic scheduling algorithm where each process is allocated a number of lottery tickets determining the possibility of process when to use the CPU. At each schedule point, a lottery is held and the process in the ready queue with the winning ticket gets the CPU utilization. Unlike priority scheduling, every job has equal chance of being represented to the processors. Lottery scheduling does not suffer from starvation.

A technical problem appears when $\mathrm{N}$ is very large, and because of the congestion in processing occurs, many processes have to wait until they are called in random manner. If all of sudden the system collapses due to failure of power supply or maintenance problem, technical faults or any other unavoidable reasons, the system manager has to look for backup management. His problem at this juncture is to know how much time requires further for finishing up the remaining processes. These predictions are uncertain and require probability mechanism to resolve. This paper takes such problem and presents two estimation methods for predicting the possible time interval required for processing the remaining jobs.

Shukla and Jain [12] discussed multiprocessor environment, for usual lottery scheduling procedures in order to obtain ready queue time estimate. A method of estimation is suggested by authors and they computed the predicted time intervals. Shukla et al. [8] studied similar problem using systematic lottery scheduling scheme in order to improve upon the prediction of ready queue processing time. Shukla et al. [11] discussed similar problem when processes are grouped according to some criteria in different queues. Shukla et al. [10] introduced size based priority scheme for the ready queue time length prediction and have shown that it is better than usual lottery scheduling in terms of confidence interval for estimates. This paper present E-F-T estimation method for such estimation and compares the two estimation procedures.

\section{A REVIEW}

Cochran [2] contains an introduction to the methods of sampling theory with application over multiple data. David [3] extended lottery scheduling, a proportional share resource management algorithm to provide the performance assurance present in traditional non real time process scheduler. Dynamic tickets were incorporated into a lottery scheduler to improve the interactive response time and to reduce kernel lock contention. Raz et al. [4] presented procedure of deciding priorities among jobs by maintaining fairness in selection procedure. Shukla and Jain [7] [9] tackled Markov Chain based study of transitions in multilevel queue scheduling. Shukla and Jain [13] performed analysis of thread scheduling and Deficit Round Robin Alternated (DRRA) scheduling algorithm using Markov Chain Model approach.

Shukla and Jain [5] suggested a stochastic model for evaluating the reaching probabilities of message flow in space-division switches. Shukla, Jain and Ojha [6] performed a task of analysis of multilevel queue scheduling with the effect of data model approach. Waldspurger [1] proposed that lottery scheduling ticket/currency framework can accommodate scheduling mechanism other than the probabilistic lottery algorithm and discussed the proportional share resource management technique. Singh and Shukla [14] discussed a family of Factor-type ratio estimator for existing population mean. In another contribution Singh and Shukla [15] derived Efficient factor type estimator for estimating the same population parameter. 
Yiping [22] developed a queuing theory model to predict system behavior and CPU queue length in Microsoft NT, Windows 2000 and derived fair share scheduling which guarantees application performance by explicitly allocating share of system resources among competing workloads. Some other useful contributions are [16], [17], [18], [19], [20], [21].

\section{PROBLEM DEFINITION}

It is common and well known idea that often the more input information provides better prediction subject to condition if information is related. Based on this thought the efficient factor type estimation technique has been introduced in order to get more precise confidence intervals compared due to Shukla et al. [14].

\section{PROCESSOR STRUCTURE AND NOTATIONS}

Let $Q_{1}, Q_{2}, Q_{3} \ldots \ldots \ldots Q_{k}$ be $k$ processors who receive intake from the ready queue containing $P_{1}, P_{2}, P_{3} \ldots \ldots \ldots P_{N}$ processes $(\mathrm{n}<\mathrm{N})$. Processes are in long, medium and short term scheduling waiting queues prepared for transferring to ready queue. When a process is blocked or suspended, it is back to respective queue. The figure 1 shows the diagram of scheduling process structured with $k$ processors.

Let;

$\mathrm{N}$ : - Total processes in the system.

n:- Sample size of randomly selected processes.

f:- Sampling fraction.

$Y_{i}$ : - CPU burst time of each $\mathrm{i}^{\text {th }}$ process $(i=1,2,3 \ldots . N)$.

$X_{i}$ : - Value of ith auxiliary variable like size of process or process priority or processes response time (time interval between arrival and processor entering).

$\bar{Y}=\frac{1}{N} \sum_{i=1}^{N} Y_{i}:-$ The mean of CPU burst time of $N$ processes in the ready queue.
$\bar{X}=\frac{1}{N} \sum_{i=1}^{N} X_{i}:-$ The mean of size of $N$ processes in the ready queue.

$C_{Y}=\frac{S_{Y}}{\bar{Y}}$ : - Coefficient of varation for population parameter Y.

$C_{X}=\frac{S_{X}}{\bar{X}}$ : - Coefficient of variation for population parameter X.

$C_{X Y}=\frac{S_{X Y}}{\bar{X} \bar{Y}}$ : - Coefficient of variation due to $\mathrm{X}$ and $\mathrm{Y}$.

\subsection{Modified Multiprocessor Lottery Scheduling}

[As Shukla, Jain and Chouwdhary[13])

Step I: When a process enters into ready queue, it is allotted a random number (in specified range).

Step II: Each processor $Q_{1}, Q_{2}, Q_{3} \ldots \ldots \ldots Q_{K}$ generates unique and uncommon random number in similar specified range stated in Step I.

Step III: Matching of both random numbers takes place between process and processor. If both random numbers are same for a process in ready queue, it is assigned to that processor.

Step IV: Processor either blocks or processes the job. It selects another process by random manner.

Step V: After when one job processed completely or partially processors generate time consumed in processing as $y_{i}$ (Time by $j^{\text {th }}$ processor) where $(j=1,2,3 \ldots \ldots . K)$ other information available auxiliary variables $X_{i}$. 


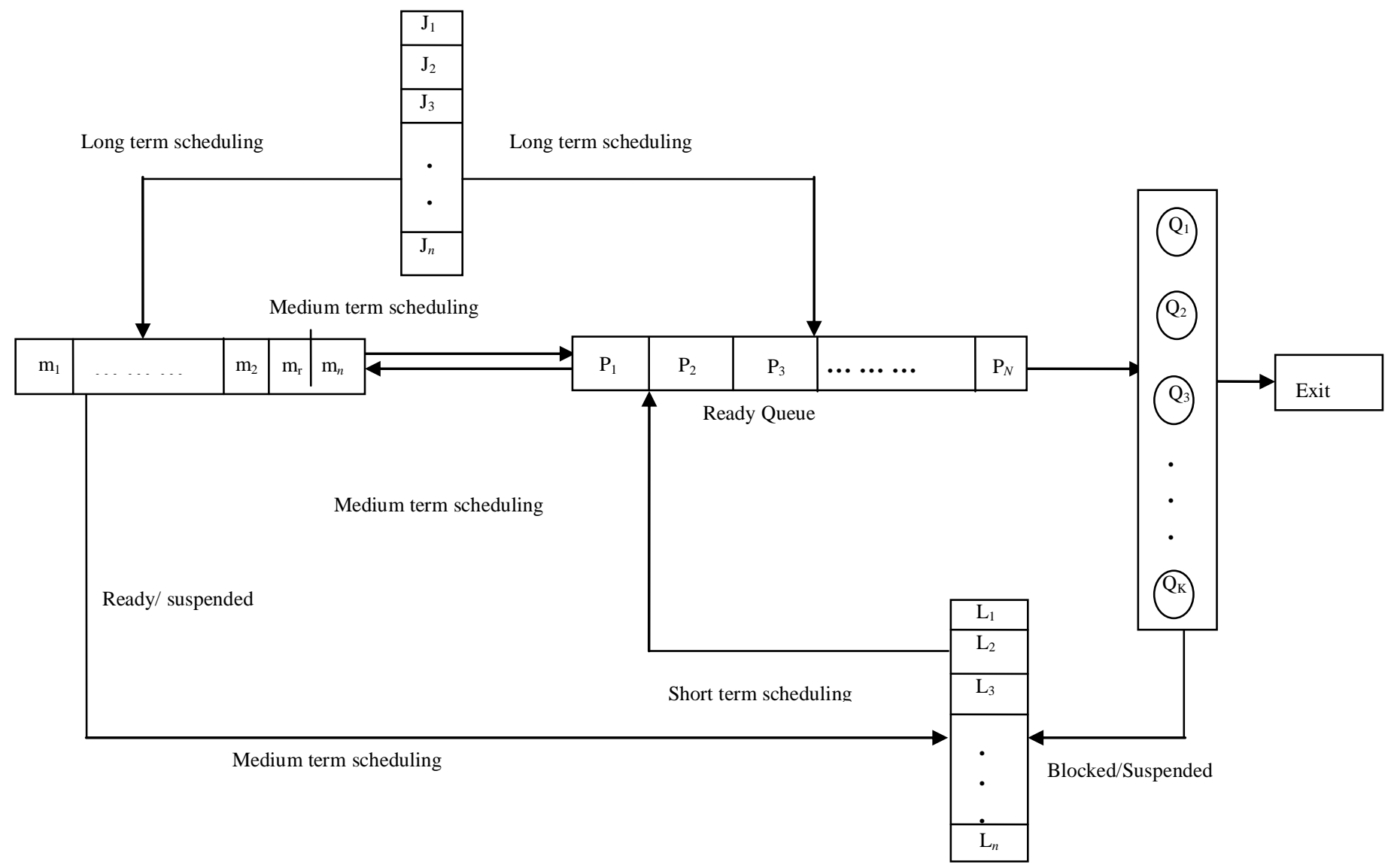

Figure 1: Ready Queue Processing Under Lottery Scheduling

\section{ESTIMATION OF READY QUEUE PROCESSING TIME (as [12])}

We know Ratio estimator: $\bar{y}_{r}=\bar{y} \bar{X} / \bar{x}$, where $\bar{y}, \bar{x}$ are sample means and $\bar{X}$ is population mean.

$$
B\left(\bar{y}_{k}\right)=\bar{Y} P V_{02} S[V-\lambda]
$$

(1)

$$
\left.M\left(\bar{y}_{k}\right)=\bar{Y} \mid V_{20}+P^{2} S^{2} V_{02}+2 P S V_{11}\right\rfloor
$$

In above expressions

$$
V_{i j}=E(\bar{y}-\bar{Y})^{i}(\bar{x}-\bar{X})^{j} / \bar{Y}^{i} \bar{X}^{j} ; \bar{Y}, \bar{X} \neq 0
$$

Consider following notations;

$$
\begin{aligned}
& A=(d-1)(d-2) \\
& B=(d-1)(d-4) \\
& C=(d-2)(d-3)(d-4)
\end{aligned}
$$

where $d$ is a constant $(0<d<\infty)$. The class of efficient factortype estimator proposed by[14] is

$$
T_{d}=\bar{y}\left[\frac{(A+C) \bar{X}+f B \bar{x}}{(A+f B) \bar{X}+C \bar{x}}\right]
$$

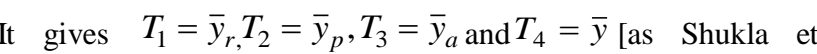
al.[14]]. The term $\bar{y}_{p}, \bar{y}_{a}$ are well known product estimator and Srivenkatramana and Tracy [19] estimator.

The fact that A, B and C can more generally be written

$$
\begin{aligned}
& A=\left(k-a_{1}\right)\left(k-a_{2}\right) \\
& B=\left(k-a_{1}\right)\left(k-b_{1}\right) \\
& C=\left(k-a_{2}\right)\left(k-b_{1}\right)\left(k-b_{2}\right) .
\end{aligned}
$$

With $b_{2}=\left(a_{2}+b_{1}\right) / 2$ has not defined in Shukla et.al [16]. They have arbitrarily chosen $a_{1}=1, a_{2}=2, b_{1}=4$ in order to define (*).However, it has been observed that if structures of A, B, C are not changed different selection of $a_{1}, a_{2}, b_{1}$ and $b_{2}=\left(a_{2}+b_{1}\right) / 2$ do not disturb the properties of the class of estimators $T_{d}$.Changes in the choice of these constants only shift the origin of $d$ to $k$. 
We define here the following one-parameter family of $E-F-T$ estimators:

$T_{k}=\bar{y}\left[\frac{(A+C) \bar{X}+f B \bar{x}_{s}}{(A+f B) \bar{X}+C \bar{x}_{s}}\right]$

(4)

$T_{k}=\bar{y}\left[\frac{(A+f B+C) \bar{X}-f B s(\bar{X}-\bar{x})}{(A+f B+C) \bar{X}-C s(\bar{X}-\bar{x})}\right]$

(5)

Where

$\bar{x}_{s}=s \bar{x}+(1-s) \bar{X} ; s=n /(N+n), 0 \leq s \leq 1 / 2$ and

$A, B, C$ and $f$ have same meaning as in (4).

\subsection{Performance Measures of $T_{k}$ [As per [14]]}

Taking large sample approximations, $\bar{y}=\bar{Y}\left(1+e_{1}\right)$ and $\bar{x}=\bar{X}\left(1+e_{2}\right)$ such that $E\left(e_{1}^{i} e_{2}^{j}=V_{i j}\right)$,

we get

$$
E\left(T_{k}\right)=\bar{Y} E\left[\left(1+e_{1}\right)\left\{\frac{(A+f B+C)+f B s e_{2}}{(A+f B+C)+C s e_{2}}\right\}\right]
$$

(6)

As it is obvious that

$$
\left|\frac{C s e_{2}}{A+f B+C}\right|<1
$$

for all choices of $\mathrm{A}, \mathrm{B}$ and $\mathrm{C}$, the bias is given by

$$
\operatorname{Bias}\left(T_{k}\right)=\bar{Y} P V_{02} S[V-\lambda]
$$

(8)

Similarly the expression for m.s.e given by

M.S.E $\left.\left(T_{k}\right)=\bar{Y} \mid V_{20}+P^{2} S^{2} V_{02}+2 P S V_{11}\right]$

(9)

Where $P=(f B s) /(A+f B+C)$ and $\lambda=C s /(A+f B+C)$

\subsection{Suggested Estimators}

Using Shukla et al. [14], we suggest two estimators as below At $d=5$, we choose one estimator in class $T_{k}$ marked as $T_{A}$

$T_{A}=\bar{y}\left[\frac{18 \bar{X}+4 f \bar{x}_{s}}{(12+4 f) \bar{X}+6 \bar{x}_{s}}\right]$

$\operatorname{Bias}\left(T_{A}\right)=\bar{Y}\left[\frac{4 f s-6}{18+4 f}\right] V_{02} s\left[V-\frac{6 s}{18+4 f}\right]$

(11)

$\operatorname{M.S.E}\left(T_{A}\right)=\bar{Y}^{2}\left[V_{20}+\left(\frac{4 f s-6}{18+4 f}\right)^{2} s^{2} V_{02}+2\left(\frac{4 f s-6}{18+4 f}\right) s V_{11}\right] \begin{aligned} & t_{A}=(N-n) T_{A} \\ & t_{B}=(N-n) T_{B}\end{aligned}$
Similarly at $d=6$, we choose one estimator from class $T_{k}$ marked as $T_{B}$

$T_{B}=\bar{y}\left[\frac{44 \bar{X}+10 f \bar{x}_{s}}{(20+10 f) \bar{X}+24 \bar{x}_{s}}\right]$

$$
\operatorname{Bias}\left(T_{B}\right)=\bar{Y}\left[\frac{10 f s-24}{44+10 f}\right] V_{02} s\left[V-\frac{24 s}{44+10 f}\right]
$$

$\operatorname{M.S.E}\left(T_{B}\right)=\bar{Y}^{2}\left[V_{20}+\left(\frac{10 f s-24}{44+10 f}\right)^{2} s^{2} V_{02}+2\left(\frac{10 f s-24}{44+10 f}\right) s V_{11}\right]$

Where $s=n /(N+n)$

The $95 \%$ confidence interval of the estimate using $T_{A}$ and $T_{B}$ are:

$$
P\left[T_{A} \pm t_{n-1, \frac{\alpha}{2}} \sqrt{V\left(T_{A}\right)}\right]=0.95
$$

$$
P\left[T_{B} \pm t_{n-1, \frac{\alpha}{2}} \sqrt{V\left(T_{B}\right)}\right]=0.95
$$

(17)

Where

$$
V\left(T_{B}\right)=\left\lfloor M\left(T_{B}\right)-\left(B\left(T_{B}\right)^{2}\right)\right\rfloor
$$$$
\text { (18) }
$$

More explicitly, one can write $95 \%$ confidence intervals for mean estimation

$$
\begin{aligned}
& P\left[T_{A}-t_{n-1, \frac{\alpha}{2}} \sqrt{V\left(T_{A}\right)} \leq \bar{Y} \leq T_{A}+t_{n-1, \frac{\alpha}{2}} \sqrt{V\left(T_{A}\right)}\right]=0.95 \\
& P\left[T_{B}-t_{n-1, \frac{\alpha}{2}} \sqrt{V\left(T_{B}\right)} \leq \bar{Y} \leq T_{B}+t_{n-1, \frac{\alpha}{2}} \sqrt{V\left(T_{B}\right)}\right]=0.95
\end{aligned}
$$

where ${ }_{n-1, \frac{\alpha}{2}}$ is t-value at (n-1) degree of freedom and at $\alpha$ level of significance and where $T_{A}, T_{B}$ are the sample based estimates of population parameter $\bar{Y}$. These estimates $T_{A}$, $T_{B}$ are predictors for average time required to complete a process by a processor. Suppose out of $N$ processes, $\mathrm{n}$ are processed $(\mathrm{n}<\mathrm{N})$ and remaining $(\mathrm{N}-\mathrm{n})$ are still in the system when sudden collapse occurs. Then the predicted total time required for remaining jobs is; where $\overline{\mathrm{X}}=5362.63, \overline{\mathrm{Y}}=73.23$. 


\section{NUMERICAL ANALYSIS}

Consider 30 processes in ready queue at a time whose size measure $\mathrm{X}$ is also given in terms of bytes. If we assume that all the processes are processed completely in the ready queue, the CPU burst time $\mathrm{Y}$ is mentioned against them.

Table 1. Data of Ready Queue

\begin{tabular}{|c|c|c|c|c|c|}
\hline Processes & $P_{1}$ & $P_{2}$ & $P_{3}$ & $P_{4}$ & $P_{5}$ \\
\hline CPU Burst Time & 30 & 20 & 112 & 40 & 59 \\
\hline Size (X) & 210 & 897 & 312 & 171 & 461 \\
\hline Processes & & & & & \\
\hline CPU Burst Time & $P_{6}$ & $P_{7}$ & $P_{8}$ & $P_{9}$ & $P_{10}$ \\
\hline Size (X) & 60 & 33 & 43 & 101 & 69 \\
\hline Processes & 290 & 379 & 220 & 470 & 636 \\
\hline CPU Burst Time & & & & & \\
\hline Size (X) & $P_{11}$ & $P_{12}$ & $P_{13}$ & $P_{14}$ & $P_{15}$ \\
\hline Processes & 138 & 43 & 109 & 26 & 74 \\
\hline CPU Burst Time & 455 & 682 & 952 & 574 & 536 \\
\hline Size (X) & & & & & \\
\hline Processes & $P_{16}$ & $P_{17}$ & $P_{18}$ & $P_{19}$ & $P_{20}$ \\
\hline CPU Burst Time & 89 & 123 & 67 & 58 & 84 \\
\hline Size (X) & 416 & 788 & 902 & 623 & 563 \\
\hline Processes & & & & & \\
\hline CPU Burst Time & $P_{21}$ & $P_{22}$ & $P_{23}$ & $P_{24}$ & $P_{25}$ \\
\hline Size (X) & 143 & 29 & 147 & 94 & 131 \\
\hline & 111 & 341 & 775 & 913 & 745 \\
\hline & & & & & \\
\hline & $P_{26}$ & $P_{27}$ & $P_{28}$ & $P_{29}$ & $P_{30}$ \\
\hline & 79 & 46 & 59 & 72 & 22 \\
\hline & 130 & 877 & 927 & 424 & 356 \\
\hline & & & &
\end{tabular}

Table 2. Population parameters for $\mathbf{N}=\mathbf{3 0}$.

\begin{tabular}{|l|l|l|l|l|}
\hline$\overline{\mathrm{X}}=5362.63$ & $\overline{\mathrm{Y}}=73.23$ & $S_{Y}^{2}=0.27409$ & $S_{X}^{2}=0.236951$ & $S_{X Y}=0.031658$ \\
\hline
\end{tabular}

Table 3. Description of first sample $n=5$.

\begin{tabular}{|c|c|c|c|c|c|}
\hline Processes Data & \multicolumn{5}{|c|}{ Sampled Processes $(n=5)$} \\
\hline Processes & $\mathrm{P}_{9}$ & $\mathrm{P}_{18}$ & $\mathrm{P}_{30}$ & $\mathrm{P}_{24}$ & $\mathrm{P}_{13}$ \\
\hline CPU Burst Time $\left(\mathbf{Y}_{\mathbf{i}}\right)$ & 101 & 67 & 22 & 94 & 109 \\
\hline $\operatorname{Processes} \operatorname{Size}\left(\mathbf{X}_{\mathbf{i}}\right)$ & 470 & 902 & 356 & 913 & 952 \\
\hline
\end{tabular}

Table 4. Population Parameters for first sample( $n=5)$.

\begin{tabular}{|l|l|l|l|l|l|l|}
\hline $\mathrm{N}=30$ & $\mathrm{n}=5$ & $\mathrm{f}=0.1666$ & $V=0.13361$ & $V_{02}=0.03949$ & $V_{20}=0.04568$ & $V_{11}=0.005276$ \\
\hline
\end{tabular}


Table 5. Bias and MSE and confidence interval of $T_{A}$ and $T_{B}$ estimators (When $\mathbf{n = 5}$ ).

\begin{tabular}{|c|c|c|c|}
\hline $\operatorname{Bias}\left(T_{A}\right)$ & -0.0115 & $\operatorname{Bias}\left(T_{B}\right)$ & -0.0126 \\
\hline$M . S . E\left(T_{A}\right)$ & 242.8404 & $M . S . E\left(T_{B}\right)$ & 241.9289 \\
\hline $\operatorname{Var}\left(T_{A}\right)$ & 242.8403 & $\operatorname{Var}\left(T_{B}\right)$ & 241.9288 \\
\hline \hline & \multicolumn{2}{|c|}{ Estimated Confidence Interval lengths } \\
\hline Estimate $\left(T_{A}\right)$ & $95 \%$ Confidence Intervals for \\
& $\left(T_{A}\right)$ & Estimate $\left(T_{B}\right)$ & $\begin{array}{c}95 \% \text { Confidence Intervals for } \\
\left(T_{B}\right)\end{array}$ \\
\hline \hline 71.18 & $(28.90-115.42)$ & 70.50 & $(28.98-115.33)$ \\
\hline
\end{tabular}

Table 6. Description of second sample $n=10$.

\begin{tabular}{|c|c|c|c|c|c|c|c|c|c|c|c|}
\hline Processes Parameters & \multicolumn{1}{|c|}{ Sampled Processes (n=10) } \\
\hline Processes & $P_{20}$ & $P_{27}$ & $P_{11}$ & $P_{1}$ & $P_{22}$ & $P_{15}$ & $P_{23}$ & $P_{5}$ & $P_{10}$ & $P_{29}$ \\
\hline \multicolumn{10}{|l|}{} \\
\hline CPU Burst Time $\left(\mathbf{Y}_{\mathbf{i}}\right)$ & 84 & 40 & 138 & 30 & 29 & 74 & 147 & 59 & 69 & 72 \\
\hline
\end{tabular}

Table 7. Population Parameters for second sample $(n=10)$.

\begin{tabular}{|l|l|l|l|l|l|l|}
\hline $\mathrm{N}=30$ & $\mathrm{n}=10$ & $\mathrm{f}=0.3333$ & $V=0.13361$ & $V_{02}=0.015797$ & $V_{20}=0.018273$ & $V_{11}=0.002111$ \\
\hline
\end{tabular}

Table 8. Bias and MSE and confidence interval of $T_{A}$ and $T_{B}$ estimators (When $\mathbf{n = 1 0}$ ).

\begin{tabular}{|c|c|c||c|}
\hline $\operatorname{Bias}\left(T_{A}\right)$ & -0.0047 & $\operatorname{Bias}\left(T_{B}\right)$ & -0.001 \\
\hline \hline M.S.E $\left(T_{A}\right)$ & 96.7872 & $M . S . E\left(T_{B}\right)$ & 96.4893 \\
\hline $\operatorname{Var}\left(T_{A}\right)$ & 96.7871 & $\operatorname{Var}\left(T_{B}\right)$ & 96.4892 \\
\hline \multicolumn{2}{|c|}{ Estimated Confidence Interval lengths } \\
\hline Estimate $\left(T_{A}\right)$ & $\begin{array}{c}95 \% \text { Confidence Intervals for } \\
\left(T_{A}\right)\end{array}$ & $\begin{array}{c}\text { Estimate }\left(T_{B}\right) \\
\text { 95\% Confidence Intervals for } \\
\left(T_{B}\right)\end{array}$ \\
\hline \hline 147.41 & $(123.82-168.33)$ & 143.46 & $(123.86-168.30)$ \\
\hline
\end{tabular}

Table 9. Description of third sample $n=15$.

\begin{tabular}{|c|c|c|c|c|c|c|c|c|c|c|c|c|c|c|c|}
\hline Processes & \multicolumn{15}{|c|}{ Sampled Processes $(n=15)$} \\
\hline Processes & $\mathrm{P}_{15}$ & $\mathrm{P}_{23}$ & $\mathrm{P}_{5}$ & $\mathrm{P}_{10}$ & $\mathrm{P}_{29}$ & $\mathrm{P}_{30}$ & $\mathrm{P}_{6}$ & $\mathrm{P}_{17}$ & $\mathrm{P}_{25}$ & $\mathrm{P}_{15}$ & $P_{20}$ & $P_{27}$ & $P_{11}$ & $P_{1}$ & $\mathrm{P}_{22}$ \\
\hline $\begin{array}{c}\text { CPU } \\
\text { Burst } \\
\text { Time }\left(\mathbf{Y}_{\mathbf{i}}\right) \\
\end{array}$ & 74 & 147 & 59 & 69 & 72 & 22 & 60 & 123 & 131 & 74 & 84 & 40 & 138 & 30 & 29 \\
\hline $\begin{array}{c}\text { Processes } \\
\operatorname{Size}\left(\mathbf{X}_{\mathbf{i}}\right)\end{array}$ & 536 & 775 & 461 & 636 & 424 & 356 & 290 & 788 & 745 & 536 & 563 & 171 & 455 & 210 & 341 \\
\hline
\end{tabular}


Table 10.Population Parameters for third sample $(n=15)$.

\begin{tabular}{|l|l||l||l|l|l|l|}
\hline $\mathrm{N}=30$ & $\mathrm{n}=15$ & $\mathrm{f}=0.5$ & $V=0.13361$ & $V_{02}=0.007989$ & $V_{20}=0.009136$ & $V_{11}=0.001055$ \\
\hline
\end{tabular}

Table 11. Bias and MSE and confidence interval of $T_{A}$ and $T_{B}$ estimators (When $\mathbf{n = 1 5}$ ).

\begin{tabular}{|c|c|c|c|}
\hline Bias $\left(T_{A}\right)$ & -0.0017 & $\operatorname{Bias}\left(T_{B}\right)$ & -0.00261 \\
\hline M.S.E $\left(T_{A}\right)$ & 48.32569 & $M . S . E\left(T_{B}\right)$ & 48.2682 \\
\hline $\operatorname{Var}\left(T_{A}\right)$ & 48.32568 & $\operatorname{Var}\left(T_{B}\right)$ & 48.2681 \\
\hline \multicolumn{2}{|c|}{ Estimated Confidence Interval lengths } \\
\hline Estimate $\left(T_{A}\right)$ & $\begin{array}{c}\text { 95\% Confidence Intervals for } \\
\left(T_{A}\right)\end{array}$ & $\begin{array}{c}\text { Estimate }\left(T_{B}\right) \\
\text { (202.65-232.47) }\end{array}$ & $\begin{array}{c}\text { 95\% Confidence Intervals for } \\
\left(T_{B}\right)\end{array}$ \\
\hline 218.97 & (202.76 & $(202.65-232.46)$ \\
\hline
\end{tabular}

\section{CONCLUSION}

Two estimators $T_{A \text { and }} T_{B}$ suggested for estimation of average ready queue remaining time. The estimate by $T_{A}=71.18$ while $T_{B}=70.50$ (when $\mathrm{n}=5$ ). Both are close to true value. The M.S.E of $T_{B}$ (=241.93) is lesser to the M.S.E of $T_{A}(=242.84)$ for $\mathrm{n}=5$. $T_{B}$ is uniformly efficient over $T_{A}$ for all $\mathrm{n}=5,10$ and 15 due to lower M.S.E. The true values of CPU burst time lies within the range of confidence interval. It is recommended to prefer $T_{B}$ over $T_{A}$ in setup of lottery scheduling for estimation purpose.

\section{REFERENCES}

[1] Carl A. Waldspurger and William E. Weihl 1994. Lottery Scheduling a flexible proportional-share resource management. In: Proceedings of the $1^{\text {st }}$ USENIX Symposium on Operating Systems Design and Implementation (OSDI), pp.1-11.

[2] Cochran 2005. Sampling Technique, Wiley Eastern Publication, New Delhi.

[3] David Petro, Garth A. Gibson and John W. Milford 1999. Implementing Lottery Scheduling: Matching the specializations in Traditional Schedulers. In: Proceedings of the USENIX Annual Technical Conference USA, pp.6680.

[4] Raz, D., B. Itzahak and Levy H. 2004. Classes, Priorities and Fairness in Queuing Systems, Research report, Rutgers University.

[5] Shukla D., Jain, S. 2010. A Stochastic Model Approach for Reaching Probabilities of Message Flow in Space-Division Switches. International Journal of Computer Networks, Vol. 2, Issue 2, pp. 140-151.
[6] Shukla, D, Jain, Saurabh and Ojha, S. 2010. Effect of Data Model Approach for the Analysis of Multi-Level Queue Scheduling, International Journal of Advanced Networking and Applications, Vol. 2 Issue 1, pp. 419-427.

[7] Shukla, D. and Jain, S. 2007. Deadlock state study in security based multilevel queue scheduling scheme in operating system. In: Proceedings of National Conference on Network Security and Management, NCNSM-07, pp. 166-175.

[8] Shukla, D., Jain, A. 2010. Estimation of ready queue processing time under SL scheduling scheme in multiprocessor environment. International Journal of Computer Science and Security (IJCSS), Vol. 4(1), pp. 7481.

[9] Shukla,D. and Jain, S. 2009. Analysis of Thread scheduling with multiple processors under a Markov chain model, Journal of Computer Science, Vol. 3 , Issue 5, pp. 78-86.

[10] Shukla,D., Jain, A. 2011. Analysis of Ready Queue Processing Time under PPS-LS and SRS-LS scheme in Multiprocessing Environment. GESJ: Computer Science and Telecommunication, 6(26), pp. 99.

[11] Shukla,D., Jain, Anjali and Choduary, A. 2010. Estimation of ready queue processing time under Usual Group Lottery Scheduling (GLS) in Multiprocessor Environment. International Journal of Computer and Applications (IJCA), Vol. 8, No. 14, pp. 39-45.

[12] Shukla,D., Jain, Anjali and Choduary, A. 2011. Estimation of ready queue processing time under Usual Lottery Scheduling (ULS) in Multiprocessor Environment. Journal of Applied Computer Science and Mathematics (JACSM), Vol. 11, No 11, pp.58-63.

[13] Shukla,D., Jain, Saurabh and Singh S. 2008. A Markov chain model for Deficit Round Robin Alternated (DRRA) scheduling algorithm. In: Proceedings of the International Conference on Mathematics and Computer Science, ICMCS-08, pp. 52-61. 
[14] Shukla D., Singh V.K., Singh G.N. 2001. On the use of transformation in factor-type estimator, METRON International Journal of Statistics, Vol. XLV, pp. 349-361.

[15] Singh V.K., Shukla D. 1992. An efficient one-parameter family of factor-type estimator in sample surveys, METRON International Journal of Statistics, Vol. XVV, pp.139-159.

[16] Singh V.K., Shukla D. 1987. One parameter family of factor type ratio estimators, METRON International Journal of Statistics, Vol. XLV- N. 1-2, pp. 273-283.

[17] Silberschatz, A. and Galvin, P. 1999. Operating System Concepts, Ed.5, John Wiley and Sons (Asia), Inc.

[18] Singh, Daroga and Choudhary, F.S. 1986. Theory and Analysis of Sample Survey and Designs, Wiley Eastern Limited, New Delhi.

[19] Srivenkataramana, T. 1980. A dual to ratio estimator in sample survey, Biometrika, Vol. 67, pp. 199-204.

[20] Stalling, W. 2004. Operating Systems, Ed.5, Pearson Education, Singapore, Indian Edition, New Delhi.

[21] Tanenbaum, A. 2000. Operating system, Ed. 8, Prentice Hall of India, New Delhi.

[22] Yiping Ding, William Flynn 2000. Interpreting Windows NT Processor Queue Length Measurements. In: Proceedings of the $31^{\text {st }}$ Computer Measurement Group Conference, Vol. 2, pp.759-770.

\section{APPENDIX}

$$
\begin{aligned}
S_{X}^{2} & =\frac{1}{N}\left[\sum X_{i}^{2}-\frac{\left(\sum X\right)^{2}}{N}\right] \\
S_{X Y}^{2} & =\frac{1}{N}\left[\left(Y_{i}-\bar{Y}\right)\left(X_{i}-\bar{X}\right)\right] \\
S_{Y}^{2} & =\frac{1}{N}\left[\sum Y_{i}^{2}-\frac{\left(\sum Y\right)^{2}}{N}\right]
\end{aligned}
$$

Table 11: Values Of ${ }^{`} t$ 'For Given Probabilities

\begin{tabular}{|c|c|}
\hline \multirow{2}{*}{$\begin{array}{c}\text { Degree } \\
\text { of } \\
\text { freedom }\end{array}$} & $\begin{array}{c}\text { Probabilities of a deviation } \\
\text { greater than } t\end{array}$ \\
\cline { 2 - 2 } & 0.025 \\
\hline 4 & 2.776 \\
\hline 9 & 2.262 \\
\hline 14 & 2.145 \\
\hline 19 & 2.093 \\
\hline 24 & 2.064 \\
\hline
\end{tabular}

